\title{
First insights into applying the Game Transfer Phenomena framework for positive means
}

\author{
Angelica B. Ortiz de Gortari \\ University of Liège, Psychology and Neuroscience of Cognition Research Unit \\ Quartier Agora Place des Orateurs 1 (B33) 4000 Liège, Belgium \\ angelica@gametransferphenomena.com
}

\begin{abstract}
Gamers have reported that their gaming experiences are pervasive and manifest even when they are not playing, re-experiencing sensorial perceptions (e.g., seeing images, hearing sounds, voices, tactile sensations), automatic responses toward game-related cues, urges to performed activities as in the game, etc This paper proposes applying the GTP framework and what has been learned on GTP for: i) strengthening interventions using virtual technologies, ii) developing or enhancing pedagogic tools for intrusions in mental disorders, and iii) understanding underlying symptoms of psychiatry and neurological conditions, including gaming addiction or Internet Gaming Disorder. Can we apply the GTP mechanisms to develop virtual applications for positive means such as learning skills, modifying interpretation of stimuli, changing dysfunctional habits, etc.? This paper overviews GTP (e.g., GTP types and characteristics), introduces the GTP framework, outlines core factors relevant for GTP, GTP mechanisms, and game contents commonly transferred, and discusses potential applications of GTP.
\end{abstract}

Keywords: Game Transfer Phenomena, GTP, video games effects, virtual reality side-effects, virtua interventions, mental disorders, involuntary phenomena

Note: The author is beneficiary of the Marie Curie COFUND fellowship, co-funded by the University of Liège and the European Union. 